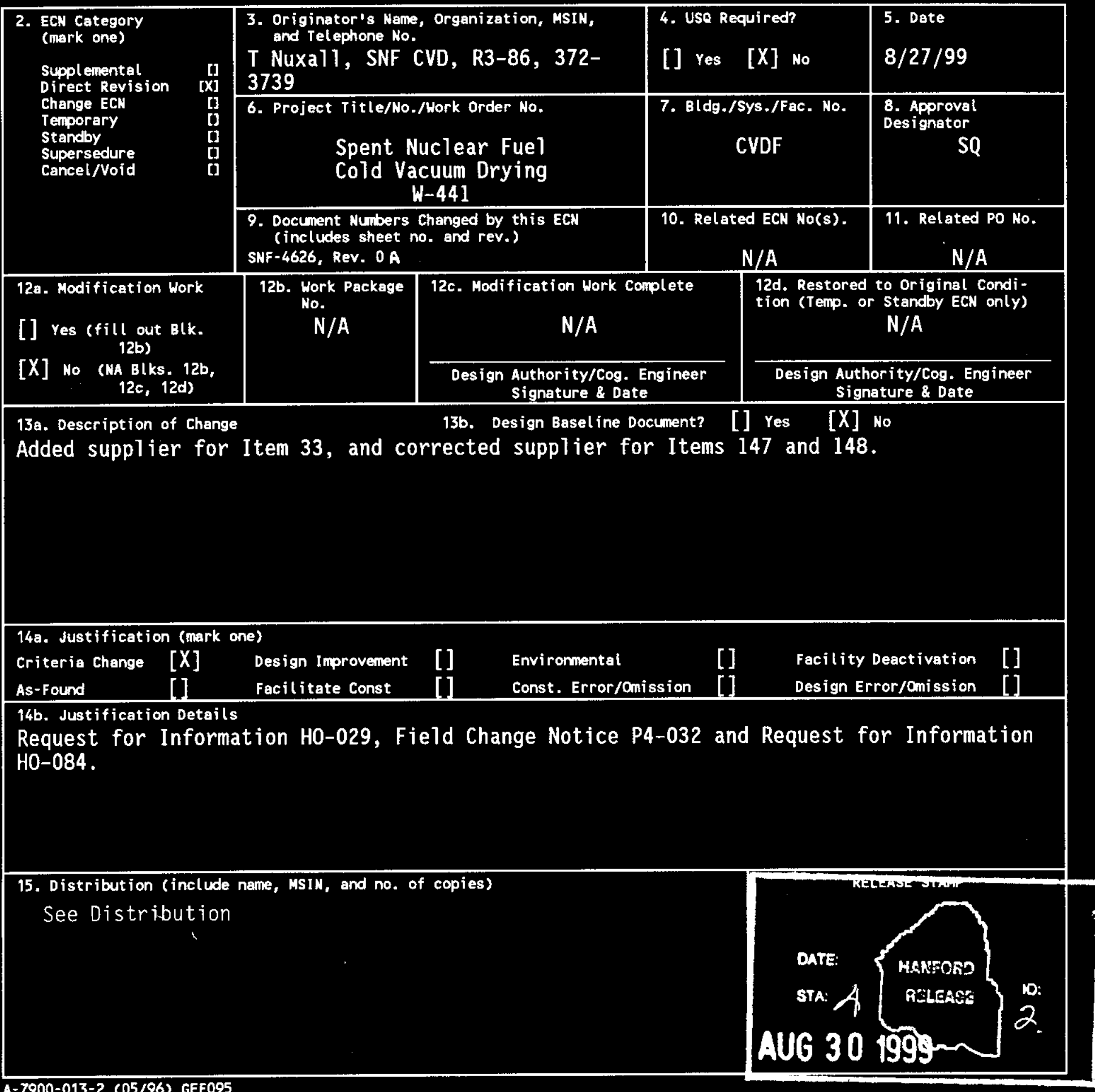




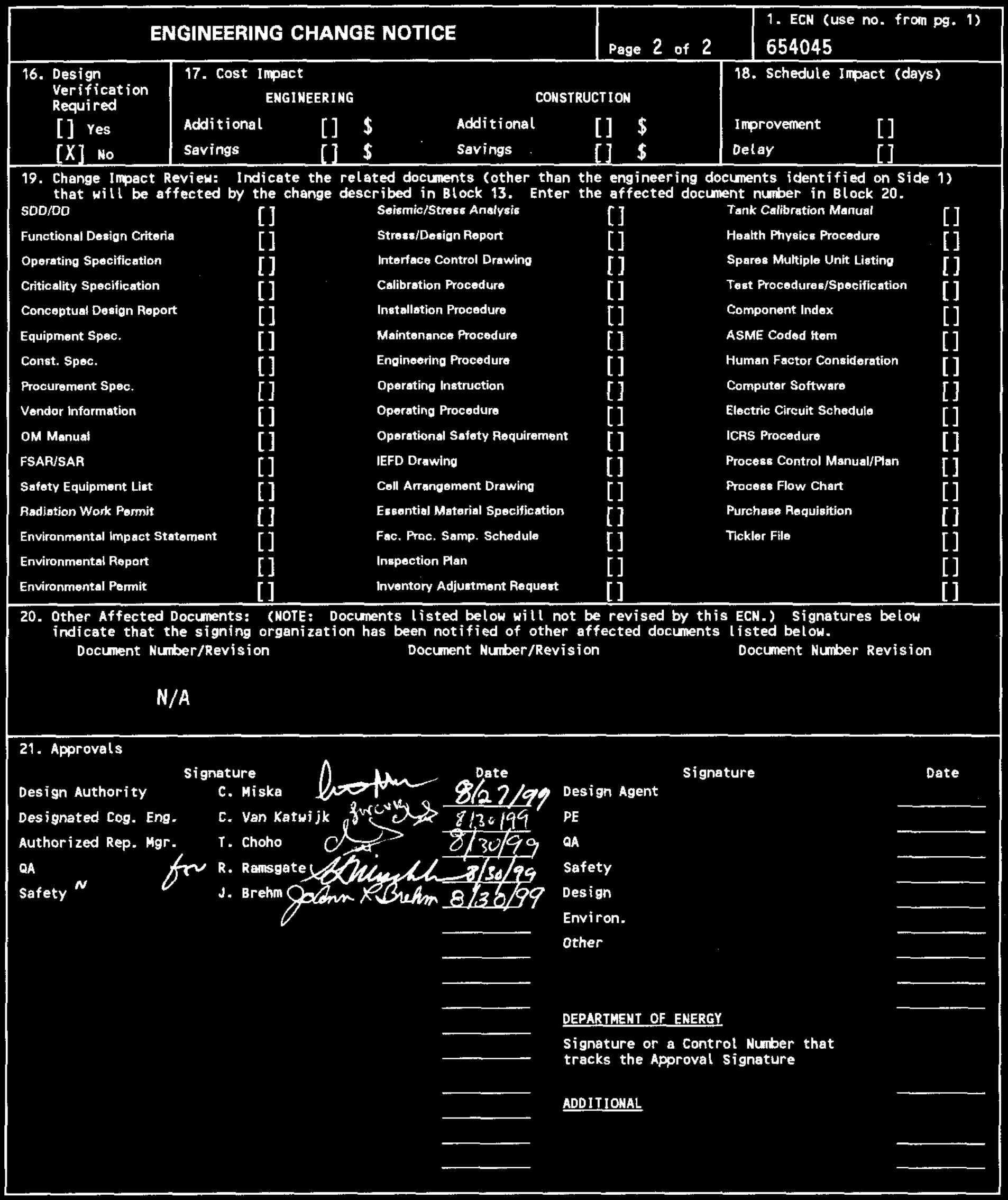




\section{PARTS FOR PROCESS HOOD ASSEMBLY PV-HOOD-1*12}

Carl Van Katwijk

Numatec Hanford, Richland, WA 99352

U.S. Department of Energy Contract DE-AC06-96RL13200

EDT/ECN: 654045

Org Code: $2 \mathrm{G} 300$

B\&R Code: 39EW40400
UC: 620

Charge Code: 105559/A000

Total Pages: 238 . 230 .

Key Words: W-441, Cold Vacuum Drying Facility (CVDF) Parts for Process Hood Assembly PV-HOOD-1*12

Abstract: W-441, Cold Vacuum Drying Facility (CVDF) Parts for Process Hood Assembly PV-HOOD-1*12

TRADEMARK DISCLAIMER Reference herein to any specific commercial product, process, or service by trade name, trademark, manufacturer, or otherwise, does not necessarily constitute or imply its endorsement, recommendation, or favoring by the United States Government or any agency thereof or its contractors or subcontractors.

Printed in the United States of America. To obtain copies of this document, contact: Document Control Services, P.O. Box 950, Mailstop H6-08, Richland WA 99352, Phone (509) 372-2420; Fax (509) 376-4989.
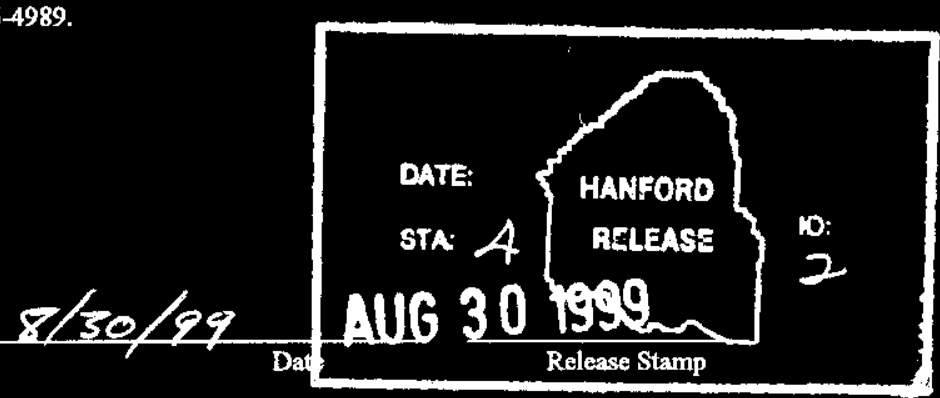

\section{Approved for Public Release}




\section{RECORD OF REVISION}

(1) Document Number
SNF-4626

(2) Titile

SNF-4626, PARTS FOR PROCESS HOOD AND ASSEMBLY PV-HOOD- 1 * 12

Change Control Record

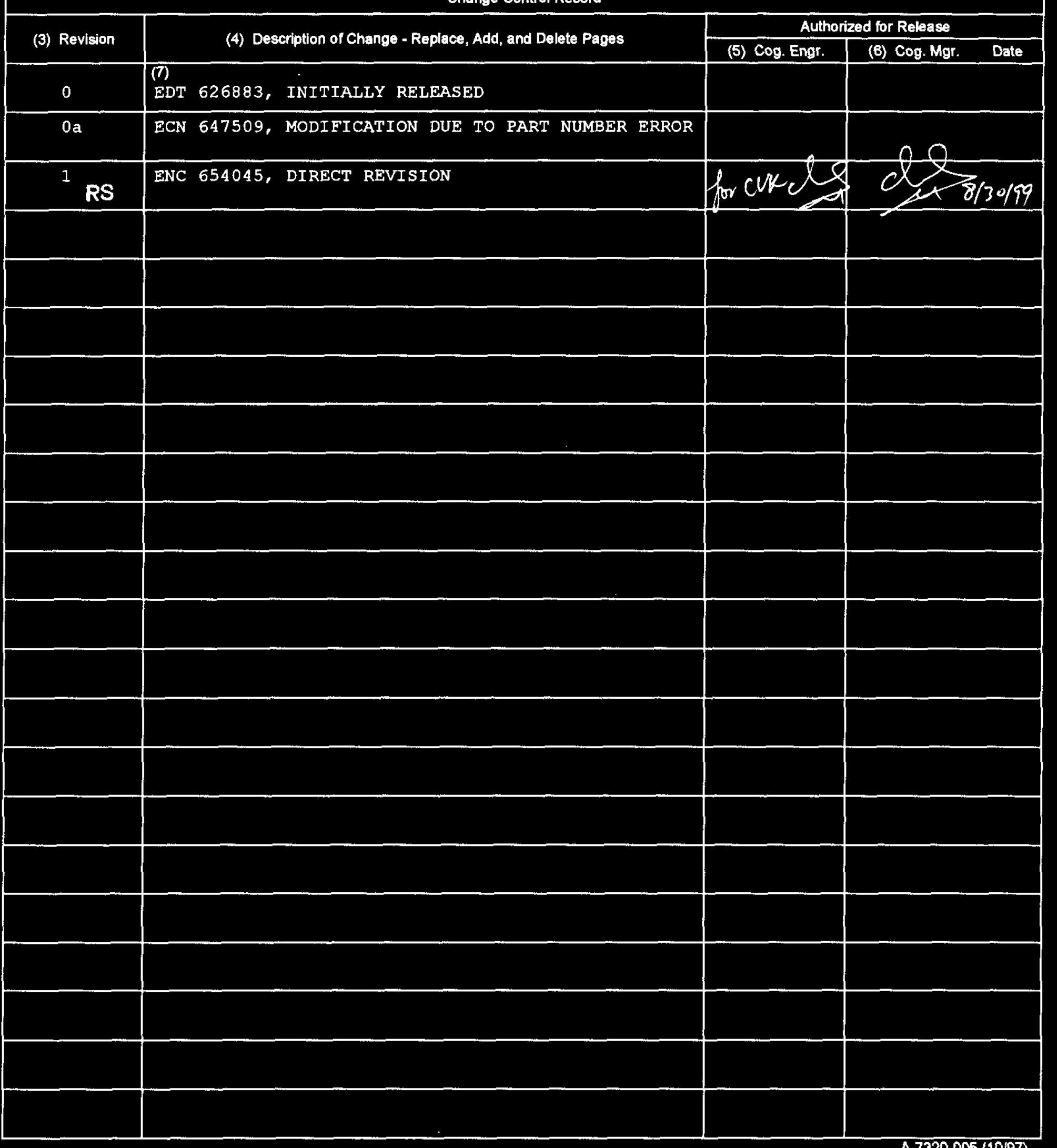




\begin{tabular}{|l|l|}
\hline Commercial Grade Item Upgrade Dedication Form & SNF-4626, Rev. 1 \\
\hline $\begin{array}{l}\text { ECN No. NA CGI No. CGI-SNF-D-30-3-P4-048 } \\
\text { Title: PARTS FOR PROCESS HOOD ASSEMBLY PV-HOOD-1*12 }\end{array}$ & Page 1 of 21 \\
\hline
\end{tabular}

\begin{tabular}{|c|c|c|c|}
\hline \multicolumn{4}{|c|}{ 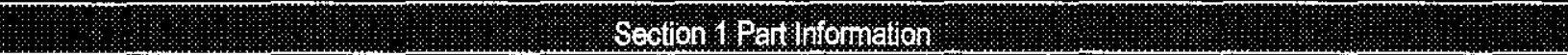 } \\
\hline Item No.: N/A & \multicolumn{2}{|l|}{ Manufacturer: } & Supplier: \\
\hline \multicolumn{2}{|l|}{ Mig. Part/Model No.: } & \multicolumn{2}{|l|}{ Supplier's P/N: } \\
\hline \multicolumn{4}{|l|}{ Part Description: } \\
\hline \multicolumn{4}{|l|}{ End Use Description: } \\
\hline \multicolumn{4}{|c|}{ Secion 2 a Componen L Litomation } \\
\hline $\begin{array}{l}\text { Equipment No.: PARTS } \\
\text { FOR PROCESS HOOD } \\
\text { ASSEMBLY PV-HOOD- } \\
1 * 12\end{array}$ & $\begin{array}{l}\text { Specification No.: W-441- } \\
\text { P4, Rev. 2; } \\
\text { Dwg.: H-1-82364 } \\
\text { with DCNs W-441- } \\
\text { H0-410, 411, 413, } \\
\text { and 416 }\end{array}$ & Manufacturer: Various & Past P.O. No.: N/A \\
\hline $\begin{array}{l}\text { Manufacturer's Part } \\
\text { Model No.: Item Numbers } \\
\text { per referenced Dwg. }\end{array}$ & \multicolumn{2}{|c|}{ Equipment Supplier (if different from manufacturer): } & Equip. Supplier's Part No.: \\
\hline $\begin{array}{l}\text { H-1-82364 Item 33; - } \\
\text { Wire Screen, 1/4" } \\
\text { Mesh X } 0.047 \text { inch } \\
\text { Wire, } 65.9 \% \text { Open }\end{array}$ & \multicolumn{2}{|c|}{ Item 33 - MeMaster-Carr } & Item $33-9266 \mathrm{~T} 2$ \\
\hline $\begin{array}{l}\text { H-1-82364, Item 143; } \\
- \text { Hose: } 9 \text { inch I.D. X } \\
20 \text { feet, Neoprene } \\
\text { Coated Flber Glass }\end{array}$ & \multicolumn{2}{|c|}{ Item 143- McMaster-Carr } & Item $143-55125 \mathrm{~K} 31$ \\
\hline $\begin{array}{l}\text { H-1-82364, Item 144; } \\
\text { - Duct Hose Clamp } 9 \\
\text { inch I.D. X } 1 / 2 \text { inch } \\
\text { width }\end{array}$ & \multicolumn{2}{|c|}{ Item 144 - McMaster-Carr } & Item $144-45955 K 75$ \\
\hline $\begin{array}{l}\text { H-1-82364, Item 146; } \\
\text { - Flex Hose, } 13 / 32 \\
\text { inch I. D., Vco female } \\
\text { swivel SST, both ends, } \\
\text { teflon hose, } 72 \text { inch } \\
\text { length }\end{array}$ & \multicolumn{2}{|l|}{ Item 146 - Swagelok } & $\begin{array}{l}\text { Item } 146 \text { - SS-TH8- } \\
\text { VF8-VF8-72 }\end{array}$ \\
\hline $\begin{array}{l}\text { H-1-82364, Item 147; } \\
\text { - Flex Hose, } 11 / 2 \\
\text { inch I. D., Male pipe } \\
\text { NPT end, SST, both } \\
\text { ends, teflon hose, Anti } \\
\text { Kink Cover, } 168 \text { inch } \\
\text { length }\end{array}$ & \multicolumn{2}{|c|}{$\begin{array}{l}\text { Item } 147 \text { - Either Meacon or Flexible } \\
\text { Components }\end{array}$} & $\begin{array}{l}\text { Item 147- 24-TLCT-03- } \\
\text { 03-S6(A)-168. }\end{array}$ \\
\hline
\end{tabular}




\begin{tabular}{|l|l|}
\hline Commercial Grade Item Upgrade Dedication Form & SNF-4626, Rev. 1 \\
\hline ECN No. NA CGI No. CGI-SNF-D-30-3-P4-048 & Page 2 of 21 \\
Title: PARTS FOR PROCESS HOOD ASSEMBLY PV-HOOD-1*12 & \\
\hline
\end{tabular}

\begin{tabular}{|l|l|l|}
\hline $\begin{array}{l}\text { H-1-82364, Item 148; } \\
\text { Flex Hose, } 1 \text { inch I. D., }\end{array}$ & $\begin{array}{l}\text { Item } 148 \text { - Either Meacon or Flexible } \\
\text { Components }\end{array}$ & $\begin{array}{l}\text { Item 148 - 16-TLCT- } \\
\text { Cend } 1 \text { inch pipe, } \\
\text { both ends, SST, teflon, }\end{array}$ \\
$\begin{array}{l}\text { Anti Kink Cover, 168 } \\
\text { inch length }\end{array}$ & \\
\hline $\begin{array}{l}\text { H-1-82364, Item 168; } \\
\text { Swivel Hoist Ring, 3/4 } \\
\text { inch, 5000 lb. capacity }\end{array}$ & Item 168 - Crosby \\
\hline
\end{tabular}

Component Description: These items are sub-assembly parts of the Process Hood, PV-HOOD-1*12. The parts are shown on W-441-P4, Rev. 2, Dwg. H-1-82364. The process hood is classed safety significant and has a safety function of radiation protection and confinement. These parts are procured from a non-qualified supplier and are being dedicated as Commercial Grade Items. Section 20 . Conmersie Avallabilizy of the liem

1. Is the Item available from a catalogue of a qualified NQA1 supplier? (coordinate with project CGI interface Engineer or BTR)

[ ] YES (go to \#2 below)

[X] NO (go to procedure step 6.3.2, proceed to dedicate Item.)

If not available from a qualified NQA1 supplier, is it available from an ISO 9000 supplier? (coordinate with project CGI interface Engineer or BTR)

[ ] YES (go to $\# 2$ below, then go to procedure step 6.3.2, proceed to dedicate ltem)

[X] NO (go to procedure step 6.3 .2 , proceed to dedicate ltem.)

2. List of Candidate qualified suppliers or ISO 9000 suppliers
company name \& type
contact name
phone

\section{N/A}

3. Recommended Procurement Strategy (coordinate with project CGI interface Engineer or BTR):

NA

\section{Secion 20 CE Deterningtion}

1. Question \#1: is the ltem subject to design or specification requirements that are unique to nuclear facilities or activities?

[ ] YES (the ltem is not commercial grade)

[X] NO (continue)

2. Question \#2: Is the ltem used in applications other than nuclear facilities or activities?

[ ] NO (the item is not commercial grade)

[X] YES (continue)

3. Question \#3: Is the Item ordered from manufacturer/supplier on the basis of specifications set forth in the published product information (e.g. manufacturer's catalog)?

[ ] NO (the ltem is not commercial grade)

[X] YES (continue)

[X] All three criteria have been satisfied. The ltem meets the definition of commercial grade. 


\begin{tabular}{|l|l|}
\hline Commercial Grade Item Upgrade Dedication Form & SNF-4626, Rev. 1 \\
\hline $\begin{array}{l}\text { ECN No. NA CGI No. CGI-SNF-D-30-3-P4-048 } \\
\text { Title: PARTS FOR PROCESS HOOD ASSEMBLY PV-HOOD-1*12 }\end{array}$ & Page 3 of 21 \\
\hline
\end{tabular}

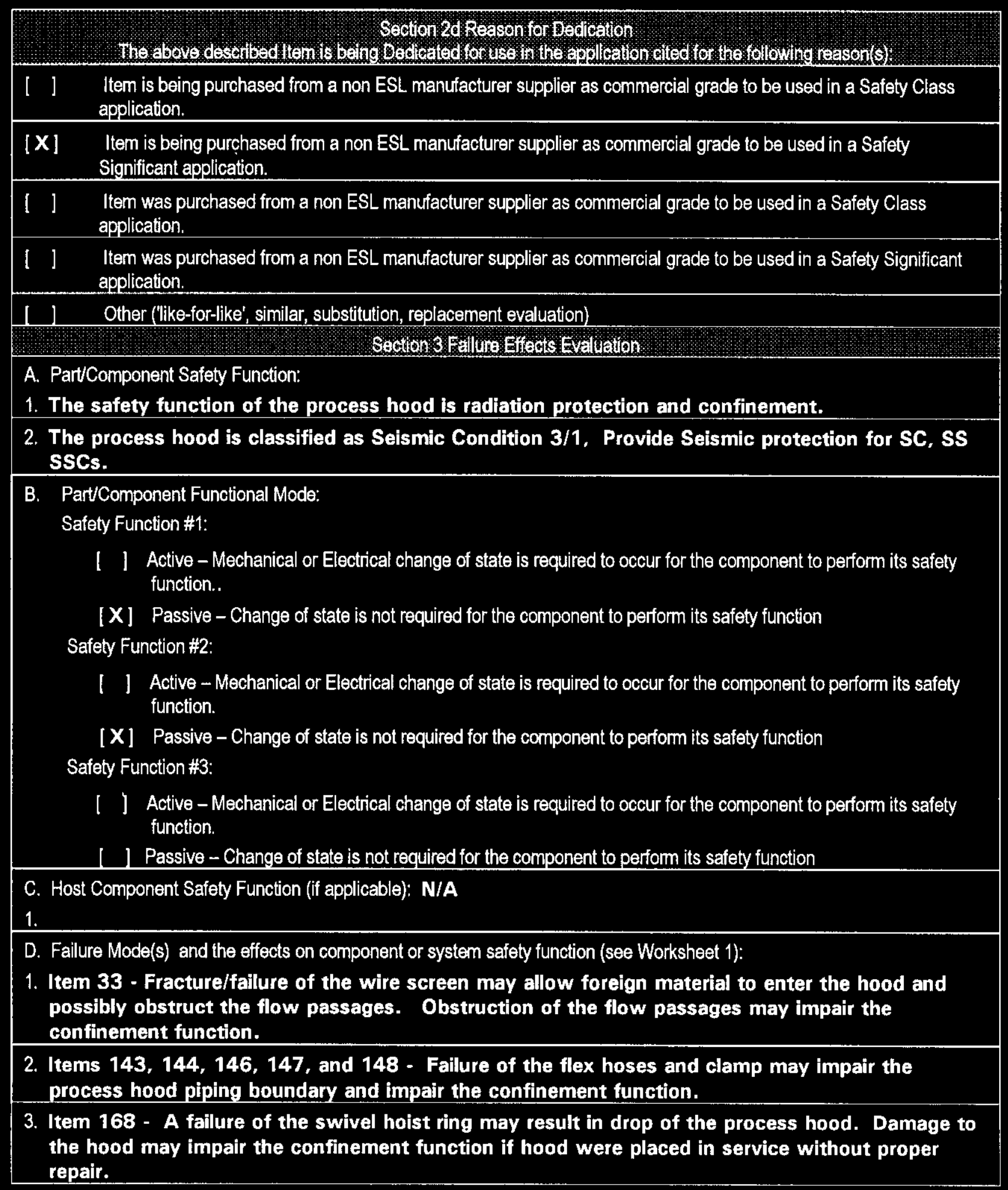




\begin{tabular}{|l|l|}
\hline Commercial Grade Item Upgrade Dedication Form & SNF-4626, Rev. 1 \\
\hline $\begin{array}{l}\text { ECN No. NA CGI No. CGI-SNF-D-30-3-P4-048 } \\
\text { Title: PARTS FOR PROCESS HOOD ASSEMBLY PV-HOOD-1*12 }\end{array}$ & Page 4 of 21 \\
\hline
\end{tabular}

\begin{tabular}{|c|c|c|c|c|c|c|}
\hline \multicolumn{2}{|l|}{$\begin{array}{l}\text { Environmental Qualification Required: } \\
\qquad \text { Yes [ ] } \\
\text { No [X] } \\
\text { Environmental Condition - None }\end{array}$} & \multicolumn{5}{|c|}{$\begin{array}{l}\text { If yes: Environmental Qualification Requirements } \\
\text { Limiting Environmental Conditions: } \\
\text { Required Safety Functions: } \\
\text { Qualification Period: }\end{array}$} \\
\hline \multicolumn{2}{|c|}{$\begin{array}{l}\text { Natural Phenomena Hazard (NPH) Design Required: } \\
\text { Yes [ ] } \\
\text { No }[\mathrm{X}] \\
\text { HNF-SD-SNF-SEL-002, Rev. } 6 \\
\text { HNF-PRO-97, Rev. } 0\end{array}$} & \multicolumn{5}{|c|}{$\begin{array}{l}\text { Condition } 3 / 1 \\
\text { Required Safety Functions: The functions for the } \\
\text { Process Hood are radiation protection and } \\
\text { confinement. Provide Seismic } 3 / 1 \text { protection for } \\
\text { SC, SS SSCs. }\end{array}$} \\
\hline \multicolumn{7}{|c|}{ 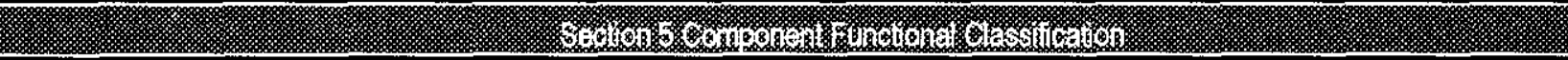 } \\
\hline \multicolumn{7}{|c|}{$\begin{array}{l}\text { [ ] Safety Class (SC) [ ] General Service (GS) [X] Safety Significant (SS) } \\
\text { If part/component classification is different from host component/system, document basis. N/A }\end{array}$} \\
\hline \multicolumn{7}{|c|}{ 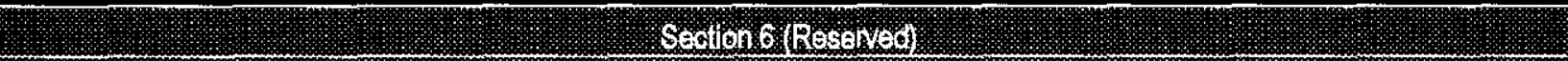 } \\
\hline \multicolumn{7}{|c|}{ SNalion } \\
\hline \multicolumn{7}{|c|}{ 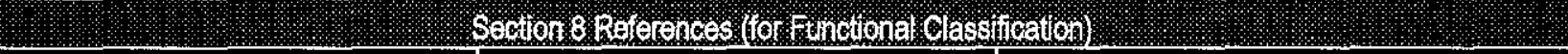 } \\
\hline $\begin{array}{l}\text { National Codes/Standards: } \\
\text { ASHRAE and ACGIH }\end{array}$ & \multicolumn{2}{|c|}{$\begin{array}{l}\text { Safety Analysis Report (SAR): HNF- } \\
\text { SD-SNF-SAR-002, Rev. } 4 \text { A }\end{array}$} & \multicolumn{4}{|c|}{$\begin{array}{l}\text { Drawings: HNF-SD-SNF-SEL-002, } \\
\text { Rev. } 6 \\
\text { Dwg.: H-1-82364 with DCNs W- } \\
441-H O-410,411,413 \text {, and } 416\end{array}$} \\
\hline \multicolumn{7}{|c|}{ Vendor Manual/Manufacturer/Supplier Information: N/A } \\
\hline \multicolumn{7}{|l|}{ Other: $\mathbf{N} / \mathbf{A}$} \\
\hline \multicolumn{7}{|c|}{ 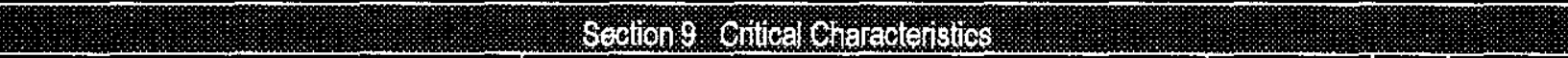 } \\
\hline $\begin{array}{l}\text { Critical Characteristics Verification } \\
\text { Document: Vendors Manuals; } \\
\text { HNF-SD-SNF-SEL-002, Rev. } 6\end{array}$ & \multicolumn{3}{|c|}{ Acceptance Criteria/Tolerances } & $\begin{array}{l}\text { Acceptance } \\
\text { Method }\end{array}$ & 10 & Function \\
\hline \multicolumn{7}{|c|}{ 1. Item Identification Critical Characteristics (necessary for reasonable assurance that the ltem delivered is the ltem specified) } \\
\hline \multicolumn{7}{|l|}{ Item 33 - Wire Screen } \\
\hline Supplier & \multicolumn{3}{|c|}{ McMaster-Carr } & 1, iN & $\mathbf{x}$ & \\
\hline Part Number & \multicolumn{3}{|c|}{$9266 T 2$} & 1, IN & $\mathbf{x}$ & \\
\hline Mesh & \multicolumn{3}{|c|}{ Nominal $1 / 4$ inch } & $1, \mathrm{IN}$ & $\mathbf{x}$ & \\
\hline Wire Diameter & \multicolumn{3}{|c|}{ Nominal 0.047 inch } & $1, \mathrm{IN}$ & $x$ & \\
\hline
\end{tabular}




\begin{tabular}{|l|l|}
\hline Commercial Grade Item Upgrade Dedication Form & sNF-4626, Rev. I \\
\hline $\begin{array}{l}\text { ECN No. NA CGI No. CCI-SNF-D-30-3-P4-048 } \\
\text { Titte: PARTS FOR PROCESS HOOD ASSEMBLY PV-HOOD-1*12 }\end{array}$ & Page 5 of 21 \\
\hline
\end{tabular}

\begin{tabular}{|c|c|c|c|c|}
\hline Item 143 - Hose & & & & \\
\hline Supplier & MoMaster-Carr & 1, IN & $\mathbf{x}$ & \\
\hline Part Number & $55125 K 31$ & 1. IN & $\mathbf{x}$ & \\
\hline I. D. & 9 inch & 1, IN & $\mathbf{x}$ & \\
\hline Length & Nominal 20 feet & 1 , IN & $\mathrm{x}$ & \\
\hline \multicolumn{5}{|l|}{ Item 144 Duct Hose Clamp } \\
\hline Supplier & McMaster-Carr & 1, IN & $x$ & \\
\hline Part Number & $45955 K 75$ & $1, \mathrm{IN}$ & $\mathbf{x}$ & \\
\hline I. D. & Nominal 9 inch & 1, IN & $x$ & \\
\hline Width & Nominal $1 / 2$ inch & 1 , IN & $\mathbf{x}$ & \\
\hline \multicolumn{5}{|l|}{ Item 146 - Flex Hose } \\
\hline Manufacturer & Swagelok & 1, IN & $x$ & \\
\hline Part Number & SS-TH8-VF8-VF8-72 & 1, IN & $\mathbf{x}$ & \\
\hline I. D. & $13 / 32$ inch & 1, IN & $\mathbf{x}$ & \\
\hline Connection & $\begin{array}{l}\text { VCO female swivel, SST, } 1 / 2 \text { inch, both } \\
\text { ends }\end{array}$ & 1, IN & $\mathbf{x}$ & \\
\hline Length & Nominal 72 inch & 1, IN & $\mathbf{x}$ & \\
\hline \multicolumn{5}{|l|}{ Item 147- Flex Hose } \\
\hline Manufacturer & Either Meacon or Flexible Components & 1, IN & $\mathbf{x}$ & \\
\hline Part Number & 24-TLCT-03-03-S6(A)-168 & 1, iN & $\mathbf{x}$ & \\
\hline I. D. & $11 / 2$ inch & 1, IN & $\mathbf{x}$ & \\
\hline Connection & $\begin{array}{l}11 / 2 \text { inch Male pipe NPT, SST, both } \\
\text { ends }\end{array}$ & 1, IN & $\mathbf{x}$ & \\
\hline Length & Nominal 168 inch & $1, \mathrm{IN}$ & $\mathbf{x}$ & \\
\hline \multicolumn{5}{|l|}{ Item 148- Flex Hose } \\
\hline Manufacturer & Either Meacon or Flexible Components & 1, IN & $\mathbf{X}$ & \\
\hline Part Number & 16-TLCT-41-41-S6A-168 & 1, IN & $\mathbf{x}$ & \\
\hline I. D. & 1 inch & $1, \mathrm{WN}$ & $\mathbf{x}$ & \\
\hline Connection & 1 inch, butt weld end, SST, both ends & 1, IN & $\mathbf{x}$ & \\
\hline Length & Nominal 168 inch & 1, IN & $\mathbf{x}$ & \\
\hline \multicolumn{5}{|l|}{ Item 168 - Swivel Hoist Ring } \\
\hline Manufacturer & Crosby & 1, IN & $\mathbf{x}$ & \\
\hline Part Number & 1016935 & 1, IN & $\mathbf{x}$ & \\
\hline Size, Ring Thickness & Nominal $3 / 4$ inch diameter & 1 , IN & $\mathbf{x}$ & \\
\hline Size, Ring Opening & Nominal 1.76 inch diameter & 1, IN & $\mathbf{x}$ & \\
\hline
\end{tabular}




\begin{tabular}{|l|l|}
\hline Commercial Grade Item Upgrade Dedication Form & SNF-4626, Rev. 1 \\
\hline $\begin{array}{l}\text { ECN No. NA CGI No. CGI-SNF-D-30-3-P4-048 } \\
\text { Title:PARTS FOR PROCESS HOOD ASSEMBLY PV-HOOD-1*12 }\end{array}$ & Page 6 of 21 \\
\hline
\end{tabular}

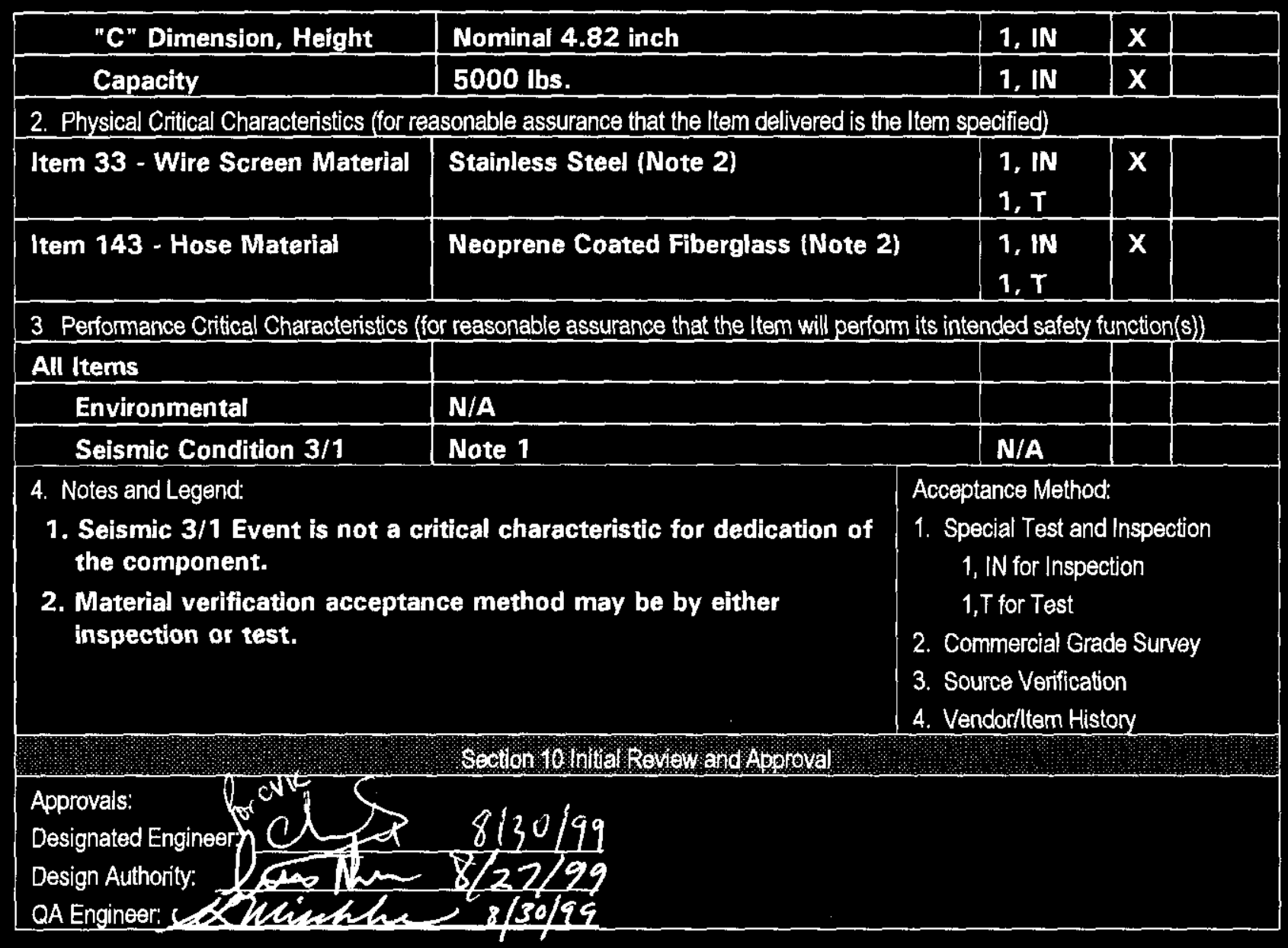




\begin{tabular}{|l|l|}
\hline Commercial Grade Item Upgrade Dedication Form & SNF-4626, Rev. 1 \\
\hline ECN No. NA CGI No. CGI-SNF-D-30-3-P4-048 & Page 7 of 21 \\
Title: PARTS FOR PROCESS HOOD ASSEMBLY PV-HOOD-1*12 & \\
\hline
\end{tabular}

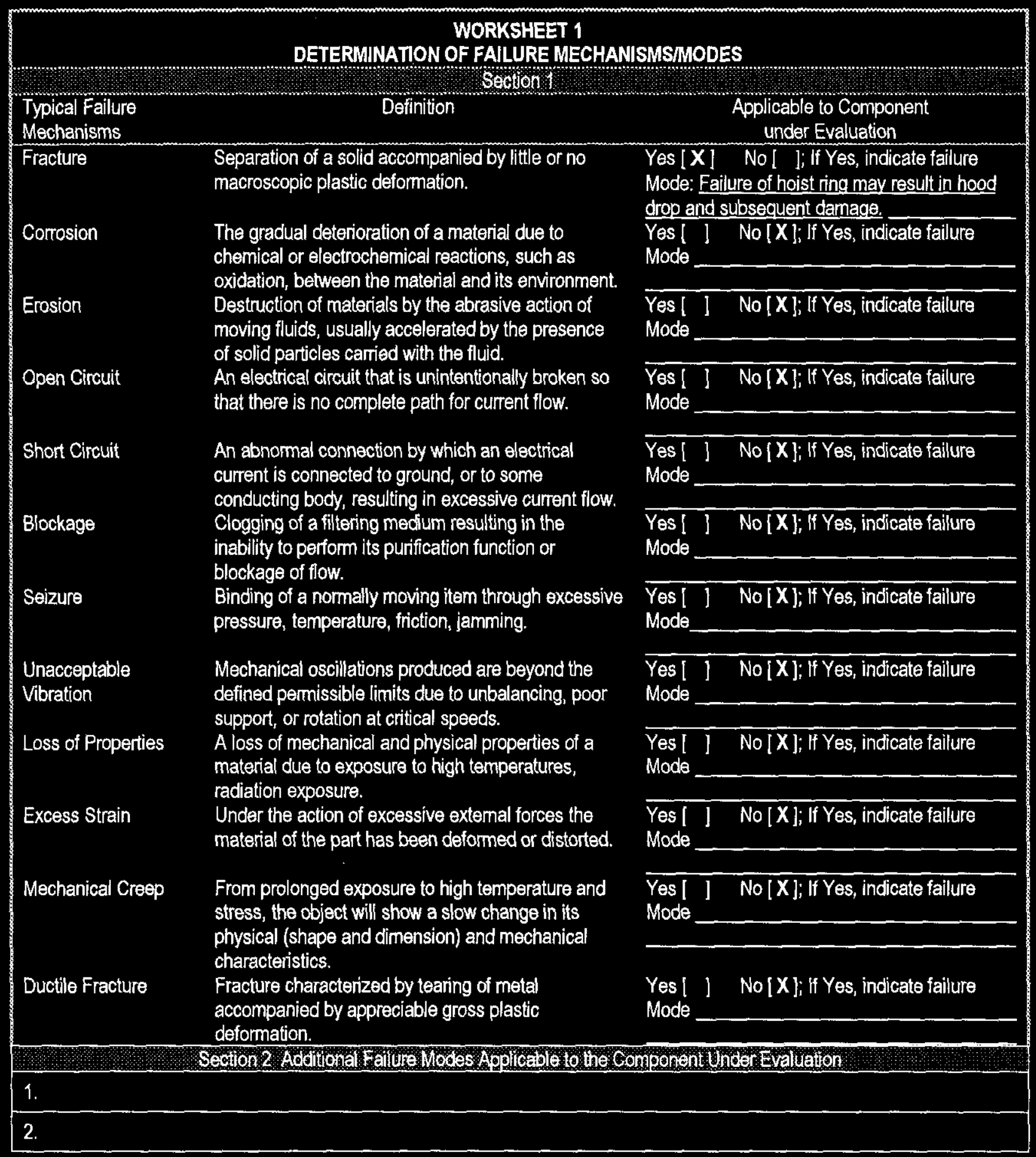




\begin{tabular}{|l|l|}
\hline Commercial Grade Item Upgrade Dedication Form & SNF-4626, Rev. 1 \\
\hline $\begin{array}{l}\text { ECN No. NA CGI No. CGI-SNF-D-30-3-P4-048 } \\
\text { Title: PARTS FOR PROCESS HOOD ASSEMBLY PV-HOOD-1*12 }\end{array}$ & Page 8 of 21 \\
\hline
\end{tabular}

\section{CHECKLIST 1}

\section{ACCEPTANCE METHOD 1}

SPECIAL TEST/INSPECTION VERIFICATION

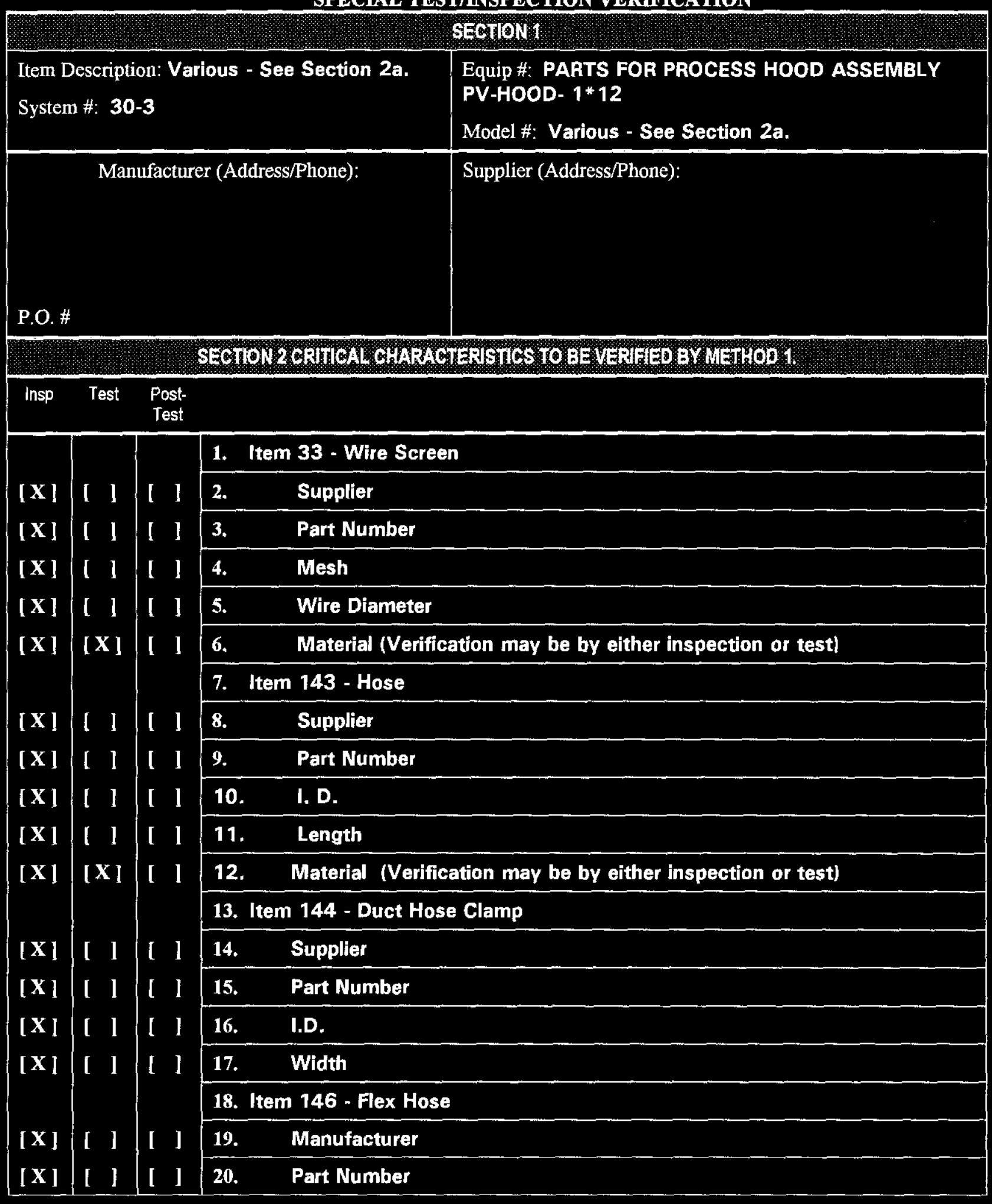




\begin{tabular}{|l|l|}
\hline Commercial Grade Item Upgrade Dedication Form & SNF-4626, Rev. 1 \\
\hline $\begin{array}{l}\text { ECN No. NA CGI No. CGI-SNF-D-30-3-P4-048 } \\
\text { Title: PARTS FOR PROCESS HOOD ASSEMBLY PV-HOOD-1*12 }\end{array}$ & Page 9 of 21 \\
\hline
\end{tabular}

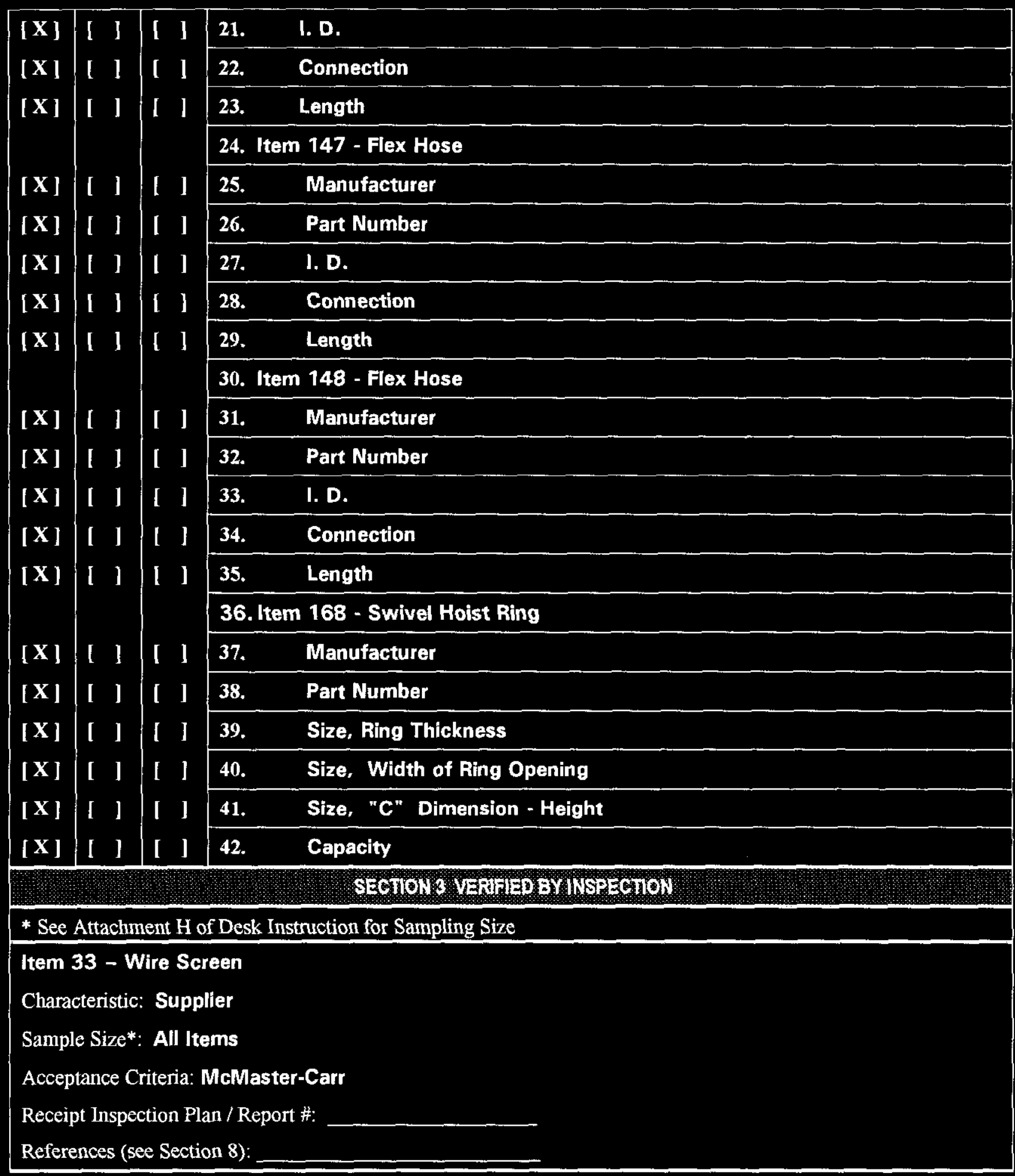




\begin{tabular}{|l|l|}
\hline Commercial Grade Item Upgrade Dedication Form & SNF-4626, Rev. 1 \\
\hline $\begin{array}{l}\text { ECN No. NA } \\
\text { Title:PARTS FOR PROCESS HOOD ASSEMBLLY PV-HOOD-1*12 }\end{array}$ & Page 10 of 21 \\
\hline
\end{tabular}

\begin{tabular}{|l|}
\hline Item 33 - Wire Screen \\
Characteristic: Part Number \\
Sample Size*: All Items \\
Acceptance Criteria: 9266 2 2 \\
Receipt Inspection Plan / Report \#: \\
References (see Section 8): \\
\hline Item 33 - Wire Screen \\
Characteristic: Mesh \\
Sample Size*: All Items \\
Acceptance Criteria: Nominal 1/4 inch \\
Receipt Inspection Plan / Report \#: \\
References (see Section 8): \\
\hline Item 33 - Wire Screen \\
Characteristic: Wire Diameter \\
Sample Size*: All Items \\
Acceptance Criteria: Nominal 0.047 inch \\
Receipt Inspection Plan / Report \#: \\
References (see Section 8): \\
\hline Item 33 - Wire Screen \\
Characteristic: Material \\
Sample Size*: All Items \\
Acceptance Criteria: Stainless Steel \\
Receipt Inspection Plan / Report \#: \\
References (see Section 8): \\
\hline Item 143 - Hose \\
Characteristic: Supplier \\
Sample Size*: All Items \\
Acceptance Criteria: McMaster-Carr \\
Receipt Inspection Plan / Report \#: \\
References (see Section 8): \\
\hline
\end{tabular}




\begin{tabular}{|l|l|}
\hline Commercial Grade Item Upgrade Dedication Form & SNF-4626, Rev. 1 \\
\hline $\begin{array}{l}\text { ECN No. NA CGI No. CGI-SNF-D-30-3-P4-048 } \\
\text { Title: PARTS FOR PROCESS HOOD ASSEMBLY PV-HOOD-1*12 }\end{array}$ & Page 11 of 21 \\
\hline
\end{tabular}

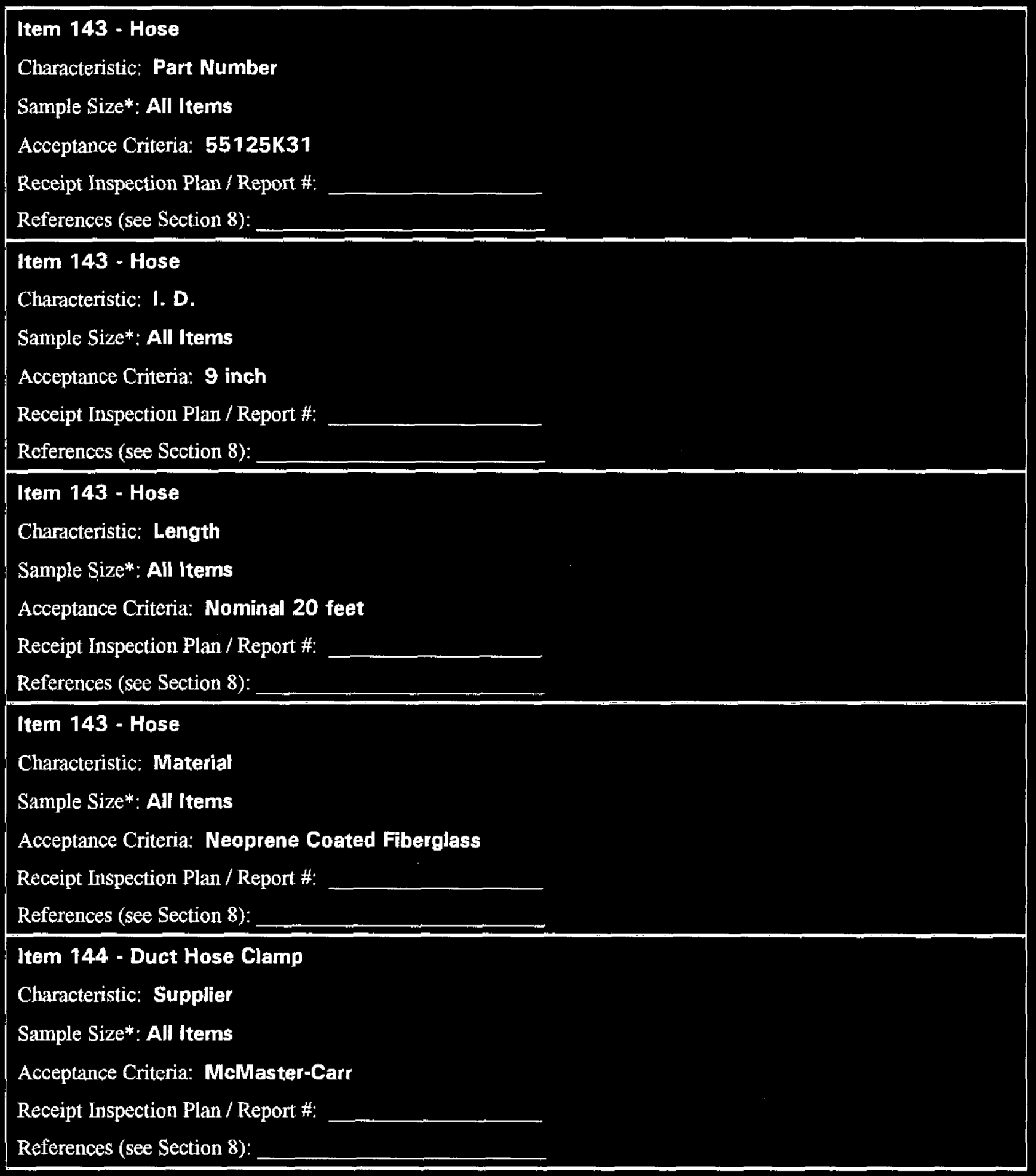




\begin{tabular}{|l|l|}
\hline Commercial Grade Item Upgrade Dedication Form & SNF-4626, Rev. 1 \\
\hline $\begin{array}{l}\text { ECN No. NA CGI No. CEI-SNF-D-30-3-P4-048 } \\
\text { Title:PARTS FOR PROCESS HOOD ASSEMBLY PV-HOOD-1*12 }\end{array}$ & Page 13 of 21 \\
\hline
\end{tabular}

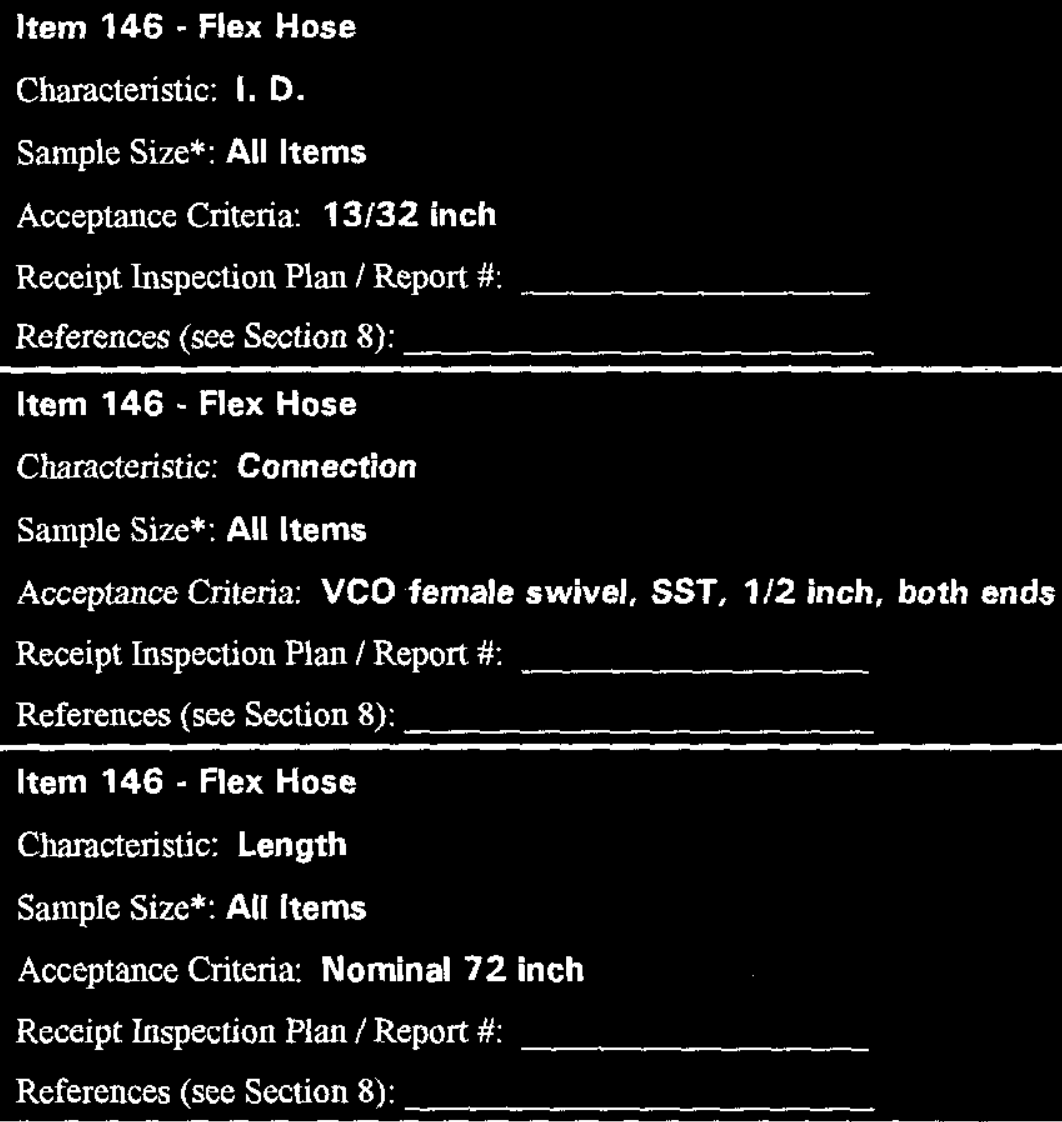




\begin{tabular}{|l|l|}
\hline Commercial Grade Item Upgrade Dedication Form & SNF-4626, Rev, 1 \\
\hline $\begin{array}{l}\text { ECN No. NA CGI No. CEI-SNF-D-30-3-P4-048 } \\
\text { Tite: PARTS FOR PROCESS HOOD ASSEMBLY PV-HOOD- } 1 * 12\end{array}$ & Page 14 of 21 \\
\hline
\end{tabular}

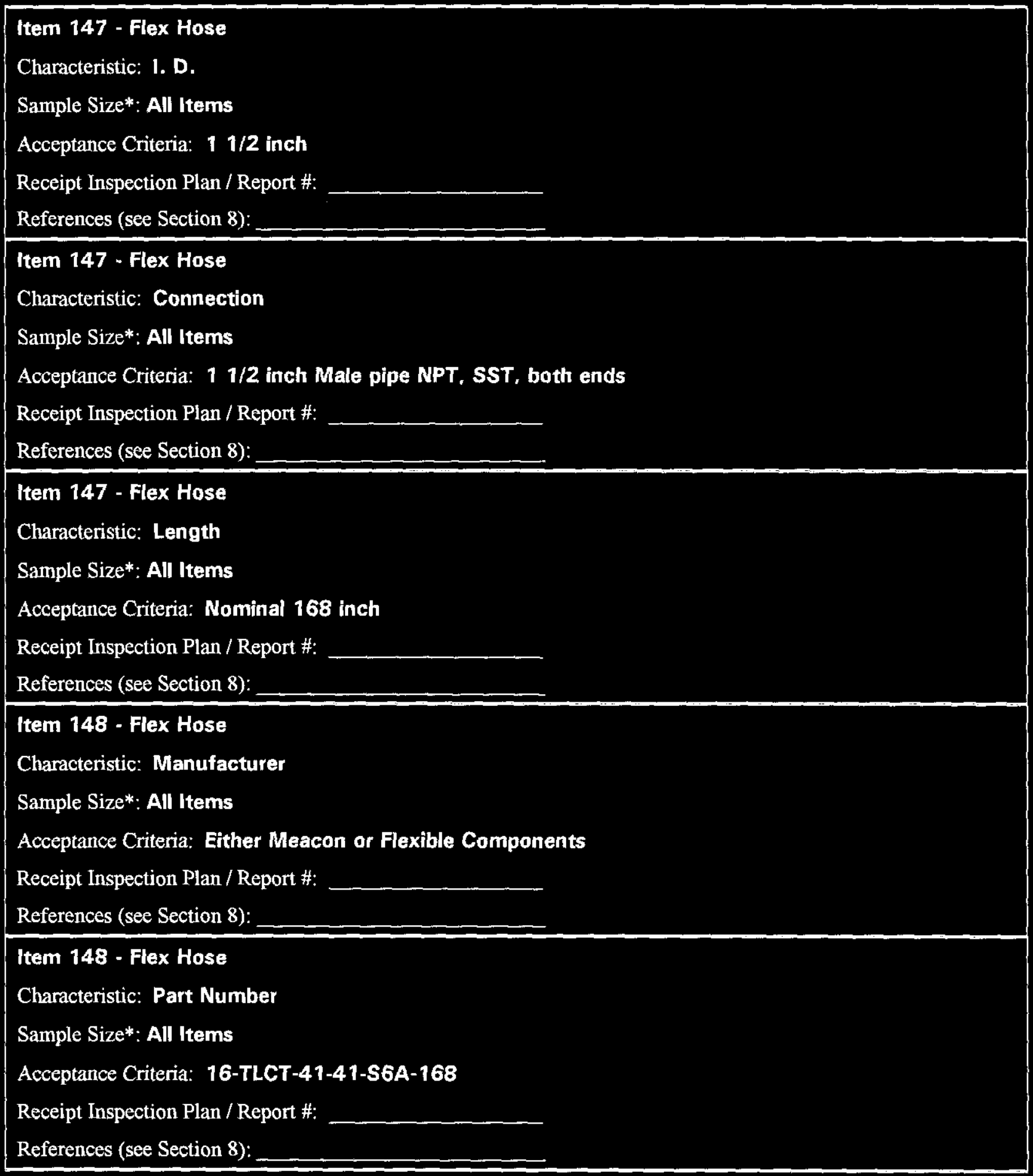




\begin{tabular}{|l|l|}
\hline Commercial Grade Item Upgrade Dedication Form & SNF-4626, Rev. 1 \\
\hline ECN No. NA COI No. CEI-SNF-D-30-3-P4-048 & Page 17 of 21 \\
Title:PARTS FOR PROCESS HOOD ASSEMBLY PV-HOOD-1*12 & \\
\hline
\end{tabular}

\section{SEGT IOW 4 WEAIFIEB BY SPECLI T TEST}

* See Attachment $\mathrm{H}$ of Desk Instruction for Sampling Size

Test To Be Performed by:

Number of Items to be Tested:

[ ] Purchaser

[ ] Supplier/Manufacturer**

Test/Inspection Location:

[ ] Other

Characteristic for Test: None

Acceptance Criteria:

Sample Size*: Normal Sampling Size

Actual Test Value:

Test Plan and Report \#: References (see Section 8):

** If Supplier/Manufacturer or Other, Refer to CGI Checklist-2 for Support Information 


\begin{tabular}{|l|l|}
\hline Commercial Grade Item Upgrade Dedication Form & SNF-4626, Rev. 1 \\
\hline ECN No. NA & Page 18 of 21 \\
Title:PARTS FOR PROCESS HOOI-SNF-D-30-3-P4-048 & \\
\hline
\end{tabular}

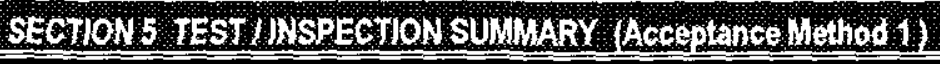

1. SUMMARY OF VERIFIED CRITICAL CHARACTERISTICS, THEIR VERIFICATION METHODS, AND RESULTS

\begin{tabular}{|c|c|c|c|c|c|c|c|c|c|c|c|}
\hline \multicolumn{4}{|c|}{ Critical Characteristics } & \multicolumn{8}{|c|}{ Verification Results } \\
\hline Citical Characteristics & Acceptance Criteria/Tolerances & 10 & Function & $\begin{array}{c}\text { Method } \\
\text { T/N } \\
\end{array}$ & $\begin{array}{c}\text { Procedure } \\
\text { or RR\# }\end{array}$ & $\begin{array}{l}\text { Check } \\
\text {-list 1D }\end{array}$ & $\begin{array}{l}\text { Number } \\
\text { Tested }\end{array}$ & $\begin{array}{c}\text { Number } \\
\text { Failed }\end{array}$ & $\begin{array}{l}\text { Verifying } \\
\text { Organization } \\
\end{array}$ & Printed Name Signature & Date \\
\hline \multicolumn{12}{|l|}{ Item 33 - Wire Screen } \\
\hline Supplier & McMaster-Carr & $x$ & & in & & & & & & & \\
\hline Part Number & $9266 \mathrm{TZ}$ & $x$ & & $\mathbb{N}$ & & & & & & & \\
\hline Mesh & Nominal $1 / 4$ inch & $x$ & & IN & & & & & & & \\
\hline Wire Diameter & Nominal 0.047 inch & $x$ & & IN & & & & & & & \\
\hline Material & Stainless Steel & $x$ & & $\mathbb{I N}, T$ & & & & & & & \\
\hline \multicolumn{12}{|l|}{ Item 143 - Hose } \\
\hline Supplier & McMaster-Carr & $x$ & & IN & & & & & & & \\
\hline Part Number & $55125 K 31$ & $x$ & & IN & & & & & & & \\
\hline I. D. & 9 inch & $x$ & & iN & & & & & & & \\
\hline Length & Nominal 20 feet & $x$ & & IN & & & & & & & \\
\hline Material & $\begin{array}{l}\text { Neoprene Coated } \\
\text { Fiberglass }\end{array}$ & $x$ & & iN, $T$ & & & & & & & \\
\hline \multicolumn{12}{|l|}{$\begin{array}{l}\text { Item } 144 \text { - Duct Hose } \\
\text { Clamp }\end{array}$} \\
\hline Supplier & McMaster-Cart & $x$ & & IN & & & & & & & \\
\hline Part Number & $45955 K 75$ & $x$ & & IN & & & & & & & \\
\hline 1. D. & Nominal 9 inch & $x$ & & IN & & & & & & & \\
\hline Width & Nominal $1 / 2$ inch & $x$ & & IN & & & & & & & \\
\hline
\end{tabular}




\begin{tabular}{|l|l|}
\hline Commercial Grade Item Upgrade Dedication Form & SNF-4626, Rev. 1 \\
\hline ECN No. NA CGI No. CGI-SNF-D-30-3-P4-048 & Page 19 of 21 \\
Title: PARTS FOR PROCESS HOOD ASSEMBLY PV-HOOD-1*12 & \\
\hline
\end{tabular}

\begin{tabular}{|c|c|c|c|c|c|c|c|c|c|c|c|}
\hline \multicolumn{4}{|c|}{ Critical Characteristics } & \multicolumn{8}{|c|}{ Verification Results } \\
\hline Critical Characteristics & Acceptance Criteria/Tolerances & $1 D$ & Function & $\begin{array}{c}\text { Method } \\
\text { TINN } \\
\end{array}$ & $\begin{array}{c}\text { Procedure } \\
\text { or RRif } \\
\end{array}$ & $\begin{array}{l}\text { Check } \\
\text {-list ID }\end{array}$ & $\begin{array}{l}\text { Number } \\
\text { Tesied } \\
\end{array}$ & $\begin{array}{c}\text { Number } \\
\text { Falled }\end{array}$ & $\begin{array}{c}\text { Verifiving } \\
\text { Organization } \\
\end{array}$ & Pinted Name Signature & Date \\
\hline \multicolumn{12}{|l|}{ Item 146 - Flex Hose } \\
\hline Manufacturer & Swagelok & $\mathbf{x}$ & & IN & & & & & & & \\
\hline Part Number & SS-TH8-VF8-VF8-72 & $x$ & & IN & & & & & & & \\
\hline I. D. & $13 / 32$ inch & $\mathrm{x}$ & & iN & & & & & & & \\
\hline Connection & $\begin{array}{l}\text { VCo female swivel, } \\
\text { SST, } 1 / 2 \text { inch, both } \\
\text { ends }\end{array}$ & $x$ & & IN & & & & & & & \\
\hline Length & Nominal 72 inch & $\mathbf{x}$ & & IN & & & & & & & \\
\hline \multicolumn{12}{|l|}{ Item 147 - Flex Hose } \\
\hline Manufacturer & $\begin{array}{l}\text { Either Meacon or } \\
\text { Flexible Components }\end{array}$ & $\mathbf{x}$ & & IN & & & & & & & \\
\hline Part Number & $\begin{array}{l}\text { 24-TLCT-03-03-SG(A)- } \\
168\end{array}$ & $\mathbf{x}$ & & IN & & & & & & & \\
\hline I. D. & $11 / 2$ inch & $x$ & & IN & & & & & & & \\
\hline Connection & $\begin{array}{l}11 / 2 \text { inch Male pipe } \\
\text { NPT, SST, both ends }\end{array}$ & $\mathbf{x}$ & & IN & & & & & & & \\
\hline Length & Nominal 168 inch & $x$ & & IN & & & & & & & \\
\hline \multicolumn{12}{|l|}{ Item 148 - Flex Hose } \\
\hline Manufacturer & $\begin{array}{l}\text { Either Meacon or } \\
\text { Flexible Components }\end{array}$ & $x$ & & IN & & & & & & & \\
\hline Part Number & $\begin{array}{l}16-T L C T-41-41-S 6 A- \\
168\end{array}$ & $x$ & & IN & & & & & & & \\
\hline I. D. & 1 inch & $x$ & & IN & & & & & & & \\
\hline Connection & $\begin{array}{l}1 \text { inch butt weld end, } \\
\text { SST, both ends }\end{array}$ & $\mathrm{x}$ & & IN & & & & & & & \\
\hline Length & Nominal 168 inch & $\mathbf{x}$ & & IN & & & & & & & \\
\hline
\end{tabular}




\begin{tabular}{|l|l|}
\hline Commercial Grade Item Upgrade Dedication Form & SNF 4626, Rev. 1 \\
\hline ECN No. NA CGI No. CGI-SNF-D-30-3-P4-048 & Page 20 of 21 \\
Title: PARTS FOR PROCESS HOOD ASSEMBLY PV-HOOD-1*12 & \\
\hline
\end{tabular}

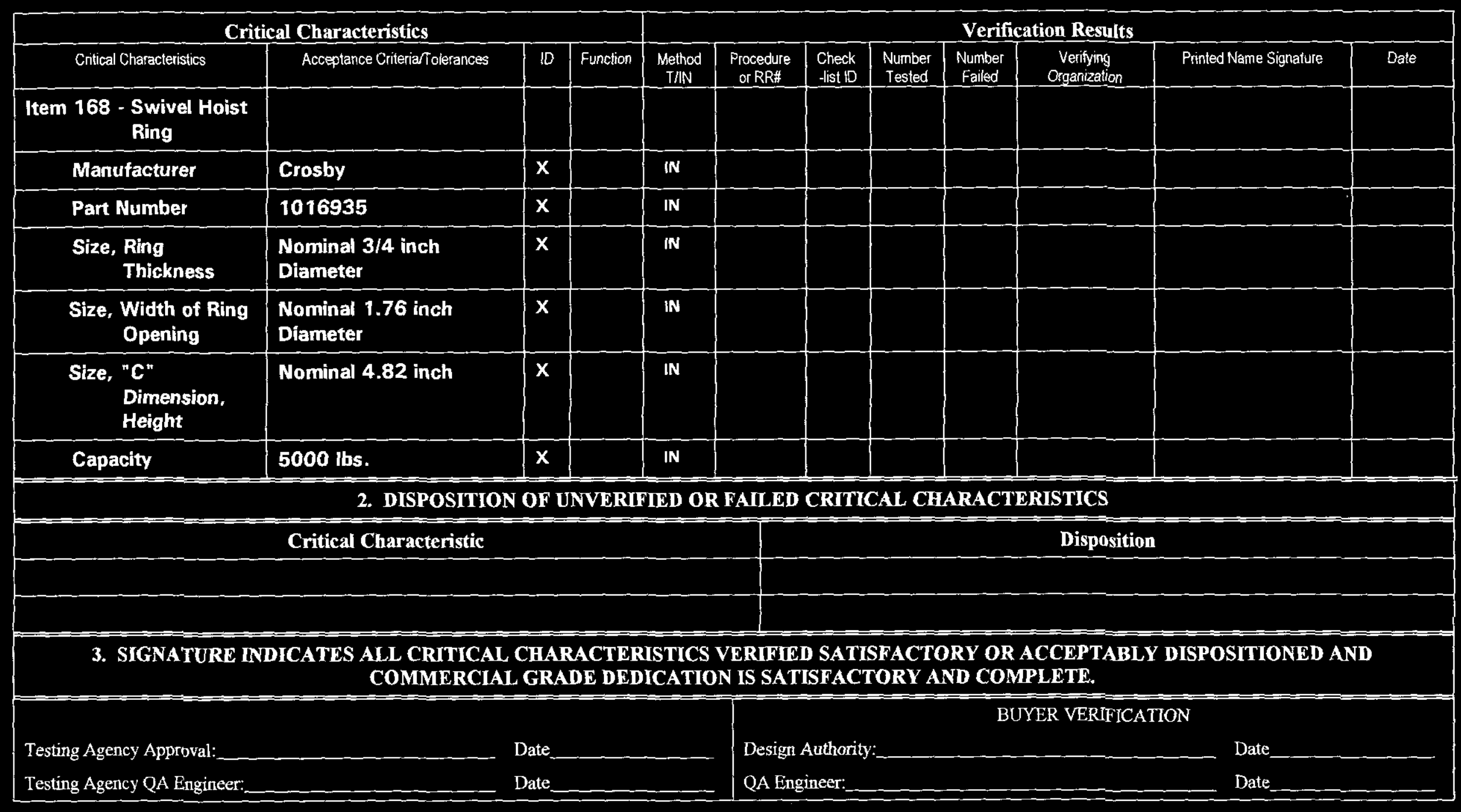




\begin{tabular}{|l|l|}
\hline Commercial Grade Item Upgrade Dedication Form & SNF-4626, Rev. 1 \\
\hline $\begin{array}{l}\text { ECN No. NA } \\
\text { Title: PARTS FOR PROCESS HOOD ASSEMBBLY PV-HOOD-1*12 }\end{array}$ & Page 21 of 21 \\
\hline
\end{tabular}

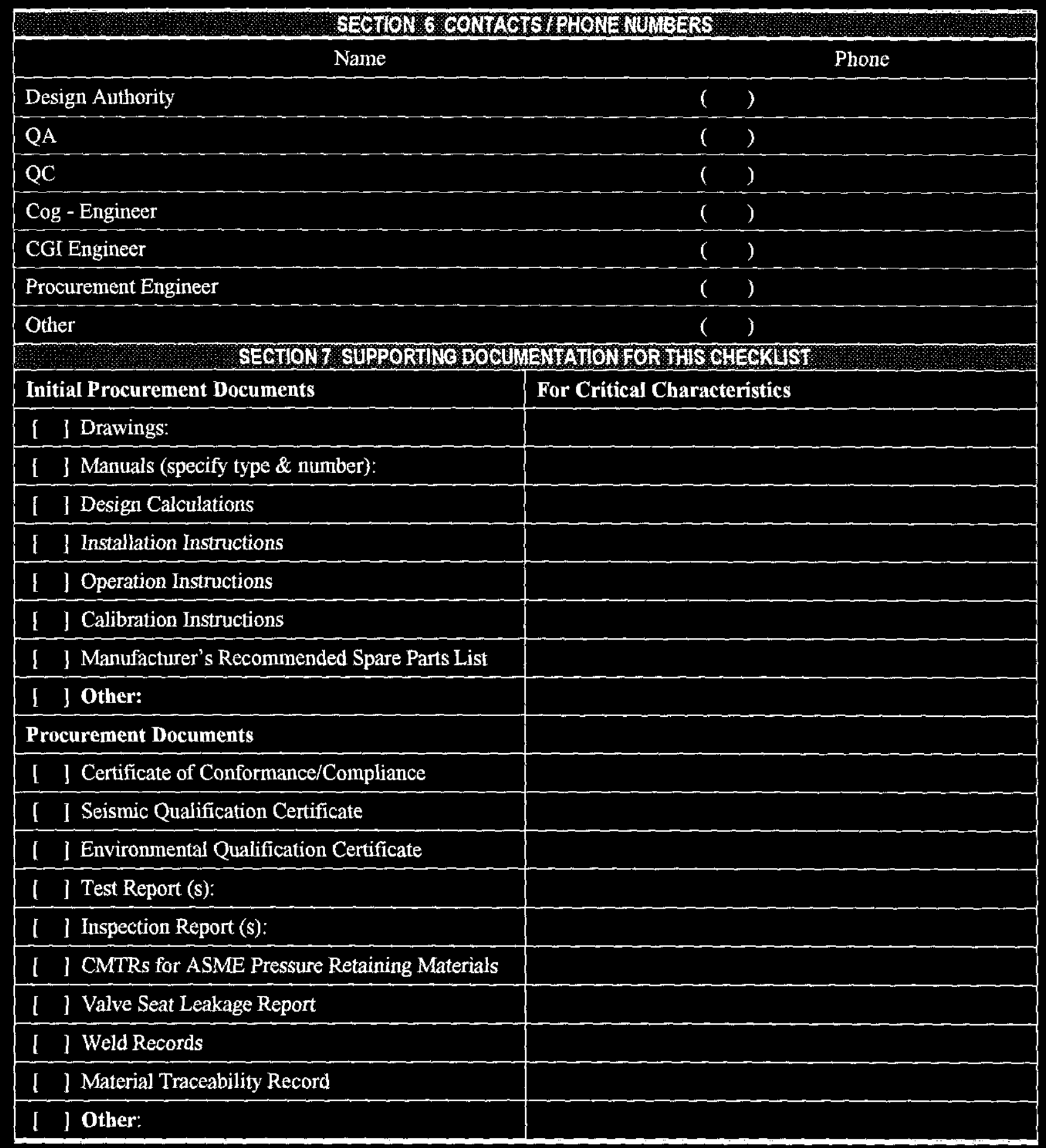




\section{DISTRIBUTION SHEET}

To

Distribution

Project ThtleNork Order

W-441, SNE-4626, PARTS FOR PROCESS HOOD ASSEMBLY PV-HOOD- $1 * 12$

Name

C. Miska/D, Whitehurst

C. Van Katwijk

A. Artzer (CVD Library)

R. Ramsgate

J. Brehm

P. Beaudet

P. Morrell (AVS)

M. Evarts (AI)

D. Marburger

SNE Startup

SNE Project Files
From

T. Nuxal1, SNE-CVD

R3-86

R3-47

R3-86

R3-86

R3-26

S8-07

$\mathrm{G} 1-50$

N1-29

R3-47

B2-64

R3-11
Page 1 of 1

Date $8 / 27 / 99$

EDT No. N/A

ECN No. 654045

\begin{tabular}{|c|c|c|c|}
\hline $\begin{array}{c}\text { Text } \\
\text { Wth All } \\
\text { Attach. }\end{array}$ & Text Only & $\begin{array}{c}\text { Attach. } \\
\text { Appendix } \\
\text { Only }\end{array}$ & $\begin{array}{c}\text { EDT/ECN } \\
\text { Only }\end{array}$ \\
\hline$x$ & & & \\
\hline$x$ & & & \\
\hline$x$ & & & \\
\hline$x$ & & & \\
\hline$x$ & & & \\
\hline$x$ & & & \\
\hline$x$ & & & \\
\hline$x$ & & & \\
\hline$x$ & & & \\
\hline$x$ & & & \\
\hline$x$ & & & \\
\hline
\end{tabular}

